\title{
Vehicular traffic modeling with greedy lane-changing and inordinate waiting
}

\author{
Damian N. Dailisan, May T. Lim* \\ National Institute of Physics, University of the Philippines Diliman, 1101 Quezon City, Philippines
}

\begin{abstract}
Lane changing and vehicular slowdowns are known to impact traffic flow. Using a modified Nagel-Schreckenberg cellular automata model for two vehicle types: blocking (e.g. cars) and non-blocking (e.g. motorcycles), we determined the thresholds at which the interplay of lane changing, random and non-random slowdowns strongly impact vehicle speeds. Lane changing improves speed with diminishing returns as vehicles opt to change lanes. At the same time, lane changing is detrimental to the overall speed when lane straddling occurs. Increasing random slowdowns beyond a critical value (in the case of motorcycles, slowdown values of $p_{\text {slow }} \approx[0.2,0.3,0.4]$ for densities $\rho=[0.20,0.15,0.10]$ respectively) can force crossover from free flowing traffic into a state where interactions between vehicles reduce the average speed.
\end{abstract}

Keywords: Computer modeling and simulation, Transportation, Monte Carlo methods statistical physics and nonlinear dynamics

\section{Introduction}

High traffic density is prevalent in urban centers, where "rush hour" traffic volume is slowly becoming the norm. Solutions to the challenge of increasing throughput despite increasing volume include real-time traffic navigation (e.g. Waze, Google Traffic) to find roads with lower occupancy, as well as optimization of sharing assignments in the nascent ride-sharing transportation network (e.g. Uber, Lyft, Grab).

The introduction of apps for ride hailing has led to a decrease in waiting time for passengers $[1,2]$ but has also introduced the unintended consequence of parked transport providers, waiting to be hailed. In cities where public transportation vehicles are privately owned, there is an additional incentive to prolong the waiting period (by waiting for price surges, or declining longer trips) to maximize profits from riders. The low cost and prevalence of on-street parking together with the increased number of vehicles due to these ride-sharing solutions ironically contribute to congestion [3].

Studies show that ride-sharing may actually be contributing to congestion, taking people away from public transit [4], and increased vacant vehicles on roads [5]. As these apps give people more control over getting to their destinations, the absence of designated stops means passengers can alight anywhere. Slowdowns caused by pickups/drop-offs have major implications in traffic flow. Changes in speed limits at slowdown sections $[6,7]$ have been shown to form traffic jams. Even small perturbations in vehicle speeds can induce congestion $[8,9]$.

Lane changing and slowdowns are behavioral quirks which have an impact on traffic flow. Cycles of bus stops and traffic signals on free-flowing traffic produce two states, which is a non-linear phenomenon [10]. This behavior combined with additional vehicles interacting on the road can be problematic in cities where public transit vehicles do not follow designated stops. Lane changing at two tollgates change the onset of jam formation [11]. The lack of lane discipline was observed to be prevalent in U-turn behavior of drivers [12]. Vehicles that perform U-turns tend to straddle multiple lanes, causing a buildup of traffic leading to the U-turn. Both lane changing and stopping are persistent elements of urban transportation modeling.

\footnotetext{
* Corresponding author

Email address: may@nip.upd.edu.ph (May T. Lim)
} 
Our goal is to determine a mapping of behavior to effective density and subsequently show the influence of effective density to overall jamming behavior. Traffic jams result in power-law distributed waiting times with no characteristic time scale [13]. The economic consequences of traffic congestion drive the challenge to model and optimize traffic flow. For example, an understanding of phase transitions [14] from free flow to congested states as a function of vehicle inflow [15] and density [7], as well as conditions leading to multiple vehicle collisions $[16,17]$, helps in traffic management and urban planning.

In this work, we focus on understanding the interplay of greedy lane changing (in an attempt for a fast trip) and frequent slowdowns (pickup/drop-off for public transportation without terminals). We use a modified Nagel-Schreckenberg (NaSch) model of two road lanes, with motorcycles and cars as primary vehicle types. We determine how greedy lane changing affects the average velocity of vehicles, by allowing only a fraction of vehicles to change lanes. We also investigate the phenomenon of slowdowns in our model and discuss phase transitions that arise from this slowdown behavior.

\section{Modeling heterogeneous traffic}

Section 2.1 discusses our implementation of the modified Nagel-Schreckenberg model used in this work and measures (Section 2.2) used to evaluate the model.

\subsection{Modified Nagel-Schreckenberg model}

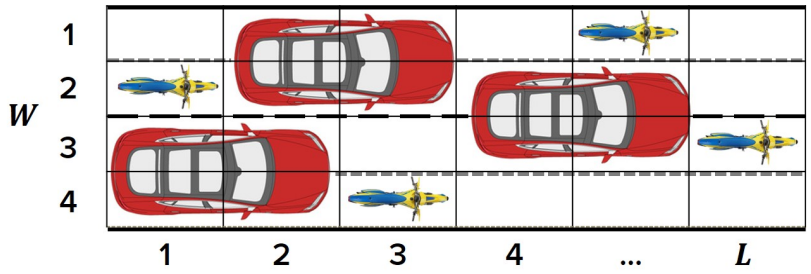

Figure 1: Diagram of two-lane road configuration of cars (2x2 cells) and motorcycles (1x1 cell). Periodic boundary conditions cause vehicles exiting the road at site $L$ to be returned to column 1 .

The model has width $W$ by length $L$ cells, with periodic boundary conditions (Fig. 1). Two types of vehicles were used, blocking and non-blocking vehicles. For real-world familiarity, blocking and non-blocking vehicles are henceforth referred to as cars and motorcycles; cars occupy 2x2 cells, while motorcycles occupy $1 \mathrm{x} 1$ cells. Vehicle states are lane $l_{i}, \operatorname{position}^{1} x_{i}$, and speed $0 \leq v_{i} \leq v_{\max }$. Cars are initially not allowed to straddle road lanes.

Realizations of the model involves assigning vehicle density $\rho$ and car fraction $\kappa$. The number of vehicles $N=\rho L W\left(\frac{\kappa}{A_{\text {car }}}+\frac{1-\kappa}{A_{\text {moto }}}\right)$, where $\rho L W$ is the number of occupied cells, $A_{\text {car }}$ and $A_{\text {moto }}$ the area occupied by cars and motorcycles, respectively. Vehicle states are updated asynchronously at each timestep $t(\delta t=1)$ following these rules:

Rule 1: Acceleration: $v_{i}=v_{i}+1$ if $v_{i}<v_{\max }$

Rule 2: Lane Change: $l_{i}=l_{i} \pm 1$ with probability $p_{\lambda}$

Rule 3: Deceleration: $v_{i}=\frac{d_{i}}{\delta t}$ if $v_{i} \delta t<d_{i}$

Rule 4: Random Slowdown: $v_{i}=v_{i}-1$ with probability $p_{\text {slow }}$

Rule 5: Forward Movement: $x_{i}=x_{i}+v_{i} \delta t$

The headway $d_{i}$ is the number of empty cells ahead of vehicle $i, p_{\text {slow }}$ represents fluctuations in driver behavior, such as slowing down to pick up passengers at arbitrary stops, or to find street parking. Vehicles may change lanes with probability $p_{\lambda} \in\{0,1\}$ with equal chances of moving left or right. Vehicle $i$ attempts to move into adjacent lanes when $v_{i}>d_{i}$ : movement is towards the lane with the largest headway among

\footnotetext{
${ }^{1}$ The position and lane of cars is taken to be the location of the driver, or the front left cell of the car.
} 
lanes within two vehicle widths away. For vehicles that have width $w>1$, like cars, the average headway of the $i^{\text {th }}$ vehicle at the $l^{\text {th }}$ lane $\bar{d}_{i, l}=\frac{1}{w} \sum_{j=0}^{w-1} d_{i, l+j}$ is considered in trying to change lanes. Vehicles may only change lanes if the target lane is empty. In the context of overtaking vehicles, Random Slowdown is skipped when a vehicle successfully changes lanes. Otherwise, the original NaSch rules $(\mathbf{1}, \mathbf{3}, \mathbf{4 , 5})$ are followed. Vehicle states are updated one vehicle at a time (asynchronously), within a single time interval $\delta t$. The sequence by which vehicle states are updated is randomized for all timesteps. The evolution of the model over time forces vehicle interactions, which give rise to complex dynamics of traffic flow.

A complete list of parameters used in our simulations, with their corresponding calibration factor and real world values, is given in Table 1 . All realizations ran for a total simulation time of $T$ timesteps, but data measurement was only started after $T_{\tau}$ timesteps so as not to include transient behavior in the data [18]. Fifty trials were done for each set of parameters $f, \rho, p_{\text {slow }}$.

Table 1: Parameters and their values used in the simulation. In this model, each cell is 2.5 meters long and 1.0 meter wide. Our simulated road is $250 \mathrm{~m}$ long and $4 \mathrm{~m}$ wide.

\begin{tabular}{cllc}
\hline Symbol & Description & Typical Values & Model Value \\
\hline$L$ & Length of the road & $100 \mathrm{~m}$ (city), 10km (highway) & $250 \mathrm{~m}$ \\
$W$ & Width of road & $10-30 \mathrm{~m}[19]$ & $4.0-5.0 \mathrm{~m}$ \\
$v_{\max }$ & Maximum speed & $20-120 \mathrm{~km} / \mathrm{h}$ (residential, highway) & $5(60 \mathrm{~km} / \mathrm{h})$ \\
$p_{\text {slow }}$ & Slowdown probability & $0.0625-0.3$ (private, PUV) [18] & $0-0.5$ \\
$p_{\lambda}$ & Lane change probability & 0,1 & $0-1$ \\
$f$ & Fraction of vehicles changing lanes & $0-1$ & $0-1$ \\
$\kappa$ & Car fraction of vehicles & $0.52[20]$ & 0,1 \\
$\rho$ & Vehicle density & $0-1($ empty, rush-hour traffic) & $0.01-0.99$ \\
$T_{\tau}$ & Transient time period & - & $2000(1500 \mathrm{~s})$ \\
$T$ & Total simulation time & - & $3000(2250 \mathrm{~s})$ \\
\hline
\end{tabular}

\subsubsection{Fraction of lane changing vehicles}

Previously, we investigated the scenario wherein all vehicles may change lanes, with a probability $p_{\lambda}$ at which they are able to successfully change lanes [21]. Here, we focus on permitting only a fraction $f$ of vehicles to change lanes with probability $p_{\lambda}=1$, with the remaining vehicles having $p_{\lambda}=0$. The fraction of vehicles that change lanes are referred to as greedy, while the remaining are non-greedy.

\subsubsection{Slowdown probability}

In the original NaSch model, the slowdown probability was added to account for random fluctuations in driving behavior. Typical values used for $p_{\text {slow }}$ ranged from 0.001 to 0.5 [22, 23], but to our knowledge, no work has been done that looks at a range of values of this parameter.

\subsection{Measurements}

From the perspective of vehicle occupants, the goal is to move as quickly as possible. We define the median velocity

$$
\tilde{v}=\operatorname{median}\left(\frac{1}{T-T_{\tau}} \sum_{t=T_{\tau}}^{T} v_{i}(t) \delta t\right),
$$

as the median value of the temporal average velocity of a vehicle. In most cases, the differences in $\tilde{v}$ for particular values of $\rho$ are small relative to the range of values observed for $\tilde{v}$. Thus, it is useful to measure the quantity $\tilde{v}-\tilde{v}_{\text {base }}$ to show subtle differences in the values of $\tilde{v}$ for different realizations of parameters. It is convenient to use $\tilde{v}_{\text {base }}$ as the case with no lane changing.

Our model used a parameter $p_{\text {slow }}$ to capture the effects of fluctuations in driving behavior. A manifestation of the effect of this parameter is in the incidence of slowdowns that occur throughout a realization of the model. A slowdown is defined as an instance wherein a vehicle $i$ has speed $v_{i}(t)<v_{i}(t-1)$. Slowdowns 
may be caused by a combination of collision avoidance via the Deceleration Rule and the fluctuations caused by $p_{\text {slow }}$. The incidence of slowdowns $N_{v_{<}}$for different realizations should be normalized against the number of vehicles $N$ and the length of time of simulation $T-T_{\tau}$.

$$
N_{v_{<}}=\frac{\operatorname{count}\left(v_{i}(t)<v_{i}(t-1)\right)}{N} \frac{1}{T-T_{\tau}}
$$

The slowdown incidence is a dimensionless metric which measures the chance that a vehicle will slow down in a timestep. When $N_{v_{<}}=1$, all vehicles always perform a slowdown for all timesteps. However, this is not possible because there is a minimum speed of $v=0$ for which no slowdowns can occur. This means that the maximum attainable value should be $N_{v_{<}}=0.5$, which is the case for vehicles alternating between speeds of 1 and 0 per timestep.

\section{Results and Discussion}

In Section 3.1, we focus on establishing the effects of the fraction parameter $f$ on the median velocity $\tilde{v}$ for the boundary cases of purely cars $(\kappa=1)$ and purely motorcycles $(\kappa=0)$. Section 3.2 shows how the theoretical value of the density dependent phase transition can be obtained from the geometrical configuration of the road and the vehicles. Section 3.3 includes a discussion on the dependence of slowdown occurences on the slowdown probability $p_{\text {slow }}$ and the resulting crossover behavior.

\subsection{Fractional lane changing}

The simulation was run for different fraction of vehicles changing lanes $f$, while using slowdown probability value $p_{\text {slow }}=0.3$. We use configurations of all motorcycles $(\kappa=0)$ or all cars $(\kappa=1)$.

\subsubsection{Diminishing returns of greedy lane changing}

To obtain a clearer view of the difference between the velocities of greedy vehicles $\left(v_{p_{\lambda}=1}=v_{\text {greedy }}\right)$ compared to non-greedy vehicles $\left(v_{p_{\lambda}=0}=v_{\text {disciplined }}\right)$, we measured the quantity $\left(\tilde{v}_{\text {greedy }}-\tilde{v}_{\text {disciplined }}\right) / \tilde{v}_{\text {all }}$. Plotting this against the road density $\rho$ (Fig. 2) shows two distinct regions. For $\rho<0.1$ and $\rho<0.15$ for motorcycles (Fig. 2a) and cars (Fig. 2b) respectively, there is no difference between the velocities of vehicles that do and do not change lanes. These values of $\rho$ fall within the free-flowing region, wherein there is enough space on the road for the vehicles to attain maximum $v$ with minimum interaction with each other.

Beyond the free-flow region, vehicles that change lanes are able to maintain higher velocities than those that do not change lanes. The relative difference between the velocities is higher when only few vehicles change lanes (low $f$ ). As the fraction of greedy vehicles increases, the advantage of lane changing vehicles over non-greedy vehicles decreases. This is observed for both motorcycles and cars, but the benefit to cars is also less than that of motorcycles, since cars can only transfer one lane at a time.

\subsubsection{Perceptions of lane changing}

In our previous model [21] wherein vehicles have a probability $p_{\lambda}$ to change lanes, it was observed that having low $p_{\lambda}$ impeded traffic flow, particularly for the case of cars wherein obstruction can occur. This effect is mitigated by ensuring that vehicles do not remain in between two lanes ( $p_{\lambda}$ close to 1 ). This work examines the interplay of successful lane changes driven by the lane changing parameter $p_{\lambda}$ and the fraction of vehicles that change lanes $f$.

Our results seem to suggest that lane changing is not as simple as anecdotal experience would show. This is illustrated in Fig. 3, where we took the difference in $\tilde{v}$ with respect to the base scenario of no lane changing $\left(f=0, p_{\lambda}=0\right)$. For the case of motorcycles, allowing lane changing introduces an increase in the median velocity of motorcycles, regardless of the fraction of lane changing vehicles $f$ and lane change probability $p_{\lambda}$ values. A different trend is observed when cars are involved. For cars, low values of $f$ have an increased average velocity if $p_{\lambda}=1$. However, with a low value of $p_{\lambda}=0.1$, lane changing is shown to reduce the average velocity of cars. 

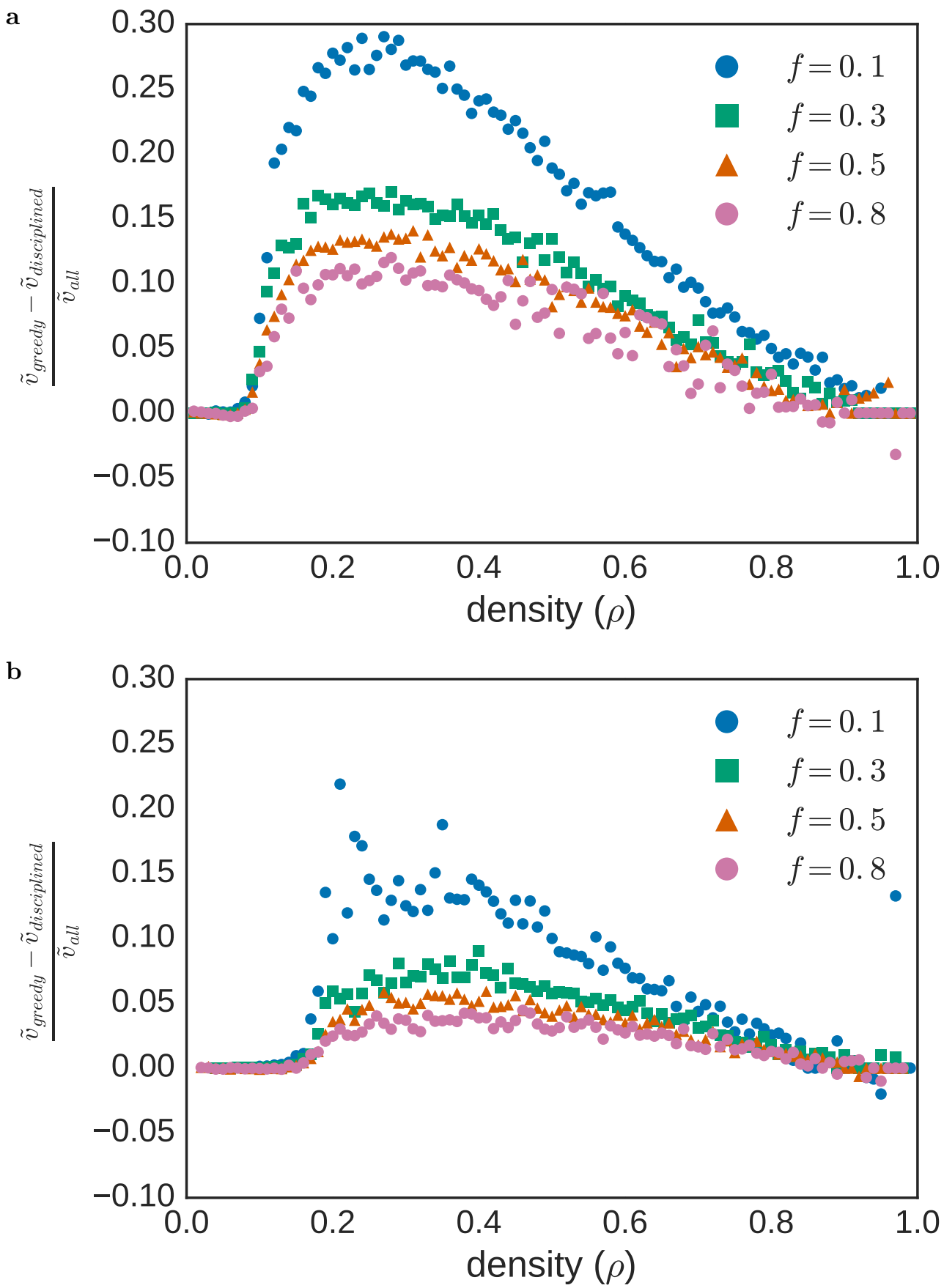

Figure 2: Difference in average velocities of greedy vs non-greedy vehicles for various values of $f=[0.1,0.3,0.5,0.8]$ for the case of (a) motorcycles (b) cars. Low values of $f$ provide the most benefit to the greedy drivers, but the improvement for cars is lower than that of for motorcycles.

Thus, we see that lane changing has the potential to improve traffic flow, provided vehicles (particularly cars) that change lanes do it in a manner which will avoid blocking several lanes at once (high lane change probability $p_{\lambda}$ ). In real world traffic however, vehicles attempt to change lanes when the road density is high, and traffic flow is already slow (i.e. impatient driving). Attempting to move into another lane while 

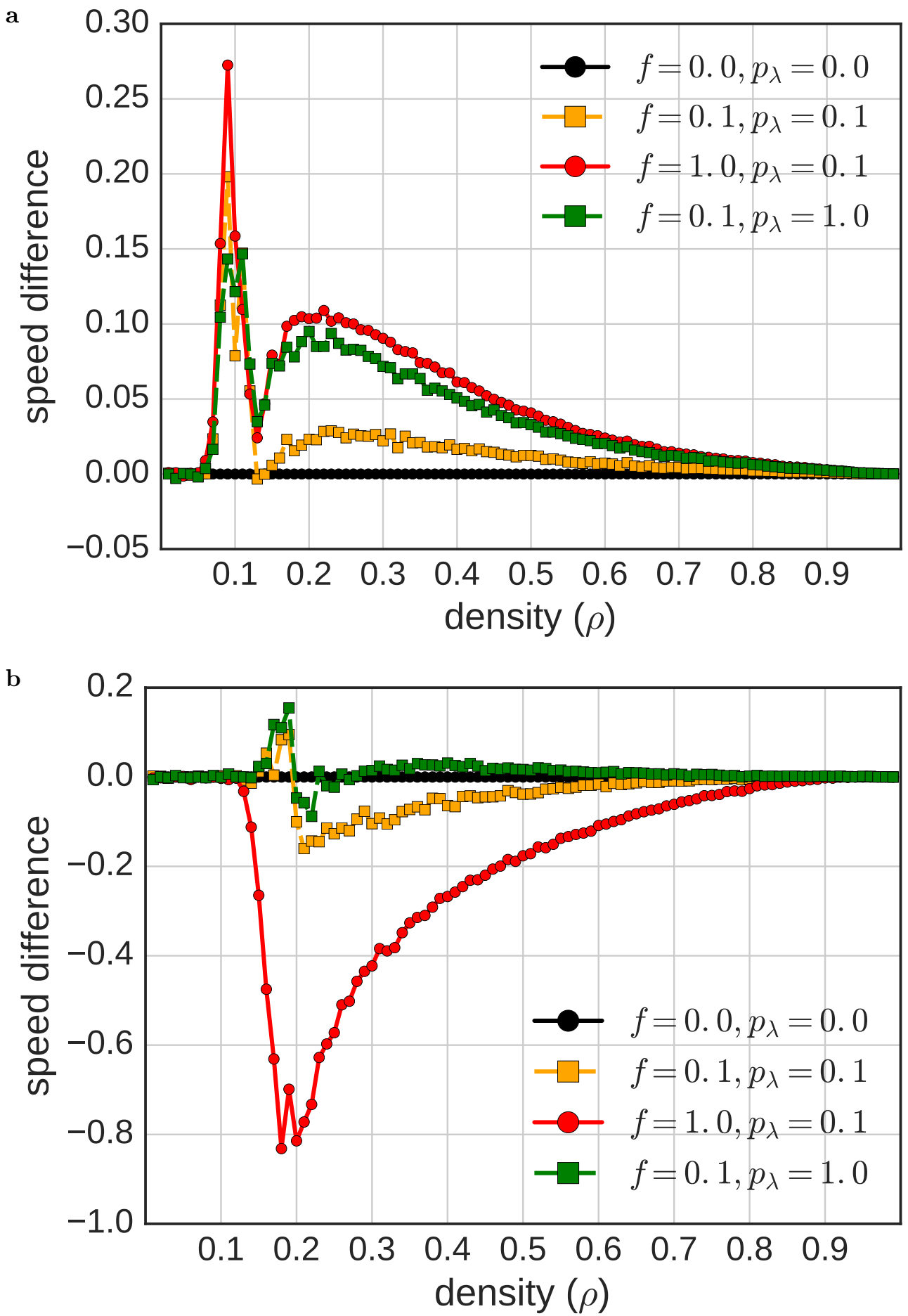

Figure 3: Median velocity comparison against no lane changing case for (a) motorcycles and (b) cars. For motorcycles, lane changing results in an increase in speed. This is not the case for cars. For the curves corresponding to low lane change probability $p_{\lambda}=0.1$ with a small fraction of vehicles changing lanes $f=0.1$ (yellow), and all vehicles changing lanes $f=1$ (red), velocity is lower than the no lane changing case. For $p_{\lambda}=1$ and low $f=0.1$ (green), we see that velocity is slightly better than the baseline case. 
taking care not to hit another vehicle effectively lowers the lane change probability, which leads to more instances that a vehicle partially occupies several lanes.

\subsection{Phase transitions}

One primary difference between a road with all cars $(\kappa=1)$ and all motorcycles $(\kappa=0)$ is that the critical densities $\rho_{\text {crit }}$ for the phase transition from a free flow state to an interacting state lies at different density values. We can see from Fig. 2 that the median velocity of motorcycles changes at $\rho_{\text {crit }} \approx 0.1$, while for cars, it occurs at $\rho_{\text {crit }} \approx 0.16$.

To find the theoretical $\rho_{\text {crit }}$, we want to find the range of values of $\rho$ wherein vehicles have minimal interactions. For a system with vehicles of length $l$, vehicles move at $v=v_{\max }$ in the free flow state. Since the only way vehicles can decelerate is through the Deceleration Rule, then it should follow that all vehicles must have headways $d_{i}>=v_{\max }$. It follows that the maximum number of vehicles $N$ that can fit into the road satisfying this condition is $N_{\text {crit }}=\frac{L}{l+v_{\max }}$. The road density that corresponds to the number of vehicles $N$ is given by $\rho_{\text {crit }}=\frac{N_{\text {crit }} l}{L}$, thus we get

$$
\rho_{\mathrm{crit}}=\frac{l}{l+v_{\max }} \text {. }
$$

The above result is a generalization of $\rho_{\text {crit }}=1 /\left(1+v_{\max }\right)[24,25]$ for the case of arbitrary vehicle length. For $l=1, v_{\max }=1, \rho_{\text {crit }}=0.5$, which is the result obtained by [24, 26]. For the values in our model, we have $v_{\max }=5$ and $l=1$ for motorcycles, $l=2$ for cars. Those values correspond to $\rho_{\text {crit }}=\frac{1}{6} \approx 0.17$ for motorcycles and $\rho_{\text {crit }}=\frac{2}{7} \approx 0.29$ for cars.

When we factor in $p_{\lambda}$, vehicles can use lane changing to avoid interactions, effectively raising $\rho_{\text {crit }}$. The random slowdown probability in turn introduces a probabilistic chance of forcing an interaction via the Deceleration Rule, effectively lowering $\rho_{\text {crit }}$.

\subsection{Random slowdown}

In this work we consider the case where all vehicles have a uniform value of $p_{\text {slow }}$, independent of the position on the road. A more realistic model would be to have a spatial dependence on $p_{\text {slow }}$ to reflect the fact that only certain regions would be loading/unloading zones. In the case of the Philippines, there are no designated stops, which is the original motivation for setting $p_{\text {slow }}$ to be position-independent.

Aside from the straightforward case of having high vehicular density $(\rho>0.8)$, it has been previously shown [27] that fluctuations in driving behavior, particularly the slowdown probability $p_{\text {slow }}$, influence traffic flow. For example, phantom jams [9] occur at densities where there are few vehicles, but the headways are sufficiently small enough to cause interactions by means of deceleration. In such cases, lane changing is an option for vehicles to mitigate the effects of these $p_{\text {slow }}$-induced random fluctuations.

Changing lanes, particularly with larger vehicles, such as cars, introduce another problem. There is a possibility that in trying to optimize its speed, a car that is changing lanes may cause vehicles behind it to slow down to accommodate the lane changing car [21]. Such was shown to be most detrimental at low $p_{\lambda}$ values. In this model, cars (width $=2$ ) take two time steps to completely change lanes. With low lane changing probabilities, cars may straddle lanes for more than two time steps. If this car slows down or stops for any reason, it effectively blocks off the rest of the road behind it.

We measure $N_{v_{<}} / \tilde{v}$, where $N_{v_{<}}$is the fraction of timesteps a vehicle slowed down. The denominator $\tilde{v}$ gives higher weight for slowdowns that occur over a slower speed, than slowdowns at a high speed. The slowdown occurrences are the result of the combination of driver fluctuations $\left(p_{\text {slow }}\right)$ and vehicle interactions (Deceleration Rule).

From Fig. 4a, we have observed a linearly increasing trend, dependent on $p_{\text {slow }}$, while the $y$-intercepts appear to depend on $\rho$. This dependence can be written in the form $\frac{N_{v_{<}}}{\tilde{v}}=m(\rho, f) p_{\text {slow }}+c(\rho)$, where $m(\rho, f)$ is the strength of the dependence of the weighted slowdown incidence on the slowdown probability, and $c(\rho)$ is the baseline measure of weighted slowdown incidences for $p_{\text {slow }}=0$. 

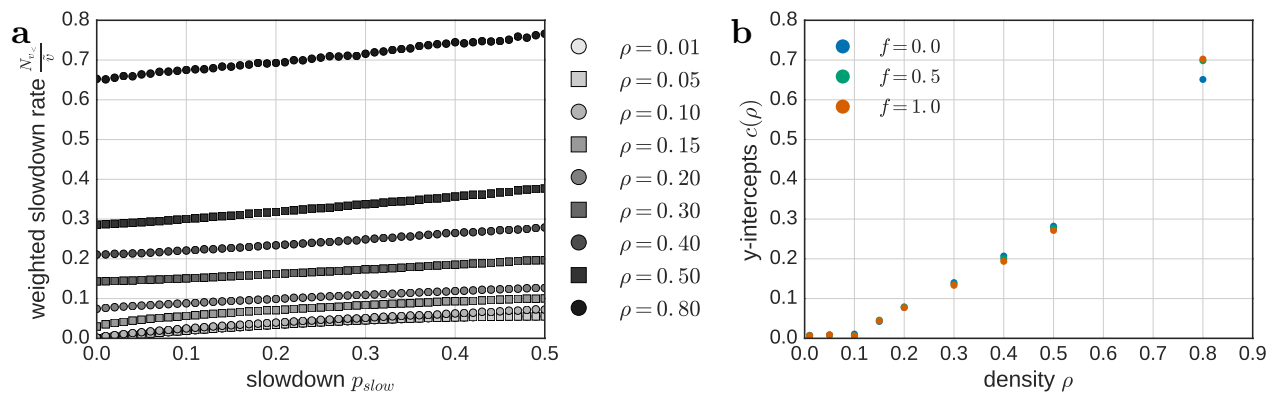

Figure 4: (a) There is a linear dependence on $p_{\text {slow }}$, with the $y$-intercepts changing with different density values. Motorcycles only $(\kappa=0)$, with no lane changing vehicles $(f=0)$. (b) $c(\rho)$ for different fractions of lane-changing motorcycles. A transition in the slope of $c(\rho)$ is seen at $\rho=0.1$; no clear dependence of $c(\rho)$ on $f$.

\subsubsection{Density dependent phase transition}

The value of the $y$-intercept $c(\rho)$ as a function of the density $\rho$ is shown in Fig. 4. A density dependent phase transition in the baseline value $c(\rho)$ is seen, but is independent of the fraction of vehicles that change lanes $f$ in the system. A corresponding phase transition from a free-flow state to an interacting state for motorcycles is also observed.

In the free-flow region $(\rho<0.1), c(\rho) \approx 0$, implying slowdowns are solely attributed to the speed fluctuations due to $p_{\text {slow }}$. In the interaction region, we see that $c(\rho) \propto \rho$, accounting for up to $87 \%$ of slowdowns (for $\rho=0.8$ ) (Fig. 4). Vehicle interactions due to increased local density is the predominant cause of vehicles slowing down.

\subsubsection{Slowdown dependent crossover}

We plot the inverse of the slowdown incidence $N_{v_{<}}$against the median velocity $1 / \tilde{v}$. We can also interpret $1 / \tilde{v}$ as the waiting time, or the average time vehicles spend on a single cell of the road. Figure 5 shows several curves (per $\rho$ ) of the slowdown rate versus average waiting time. Each point on the curve corresponds to distinct $p_{\text {slow }}$ values.

Two trends are noticeable in Fig. 5a. For low densities $\rho<0.3$ (Fig. 5a), a trend reversal (manifesting as peaks) is observed in slowdown rate as the waiting time increases for $\rho=0.1,0.15,0.2$. The monotonically decreasing trend is a signature of the road being in the interacting state. No peak is visible for the lower densities $\rho=0.01,0.05$.

We take interest in the peaks formed by the curves corresponding to $\rho=0.1,0.15,0.2$. Since each point on the curve corresponds to a particular value of the slowdown probability, we want to see how the slope slope $=\frac{\Delta N_{v}}{\Delta(1 / \tilde{v})}$ varies with $p_{\text {slow }}$. The plot of the slopes against the corresponding slowdown probability $p_{\text {slow }}$ values are shown in Fig 6 .

The peaks, which occur when the slope becomes zero, are shown to occur at different $p_{\text {slow }}$ values. For increasing $\rho$, the slope becomes zero at lower slowdown probabilities. This provides insight on the effect of $p_{\text {slow }}$ at low densities. From the plots of the slowdown incidence versus waiting time for the densities $\rho>0.3$ (Fig. 5), the monotonically decreasing trend is a signature of the road being in the interacting state. The peaks represent a transition on the correlation between the number of slowdowns and average waiting times. This crossover is also related to phase transition from a free-flow state to the interaction state.

It was shown in Sec. 3.2 that the theoretical value of $\rho_{\text {crit }}$ for motorcycles $(\kappa=0)$ lies at $\rho \approx 0.17$. However, in our simulations, we use a non zero value of the random slowdown probability. The value slowdown probability $p_{\text {slow }}=0.3$ was used particularly in Sec. 3.1 , wherein it was shown that the critical density is $\rho_{\text {crit }} \approx 0.10$. But as Fig. 6 suggests, for density $\rho=0.10$ the crossover due to slowdown probability $p_{\text {slow }}$ occurs when $p_{\text {slow }}=0.4$. This means that although the slowdown probability is associated with a transition from free flow to interaction behavior, the crossover point when the slope is zero is not the same as the phase transition due to density. 

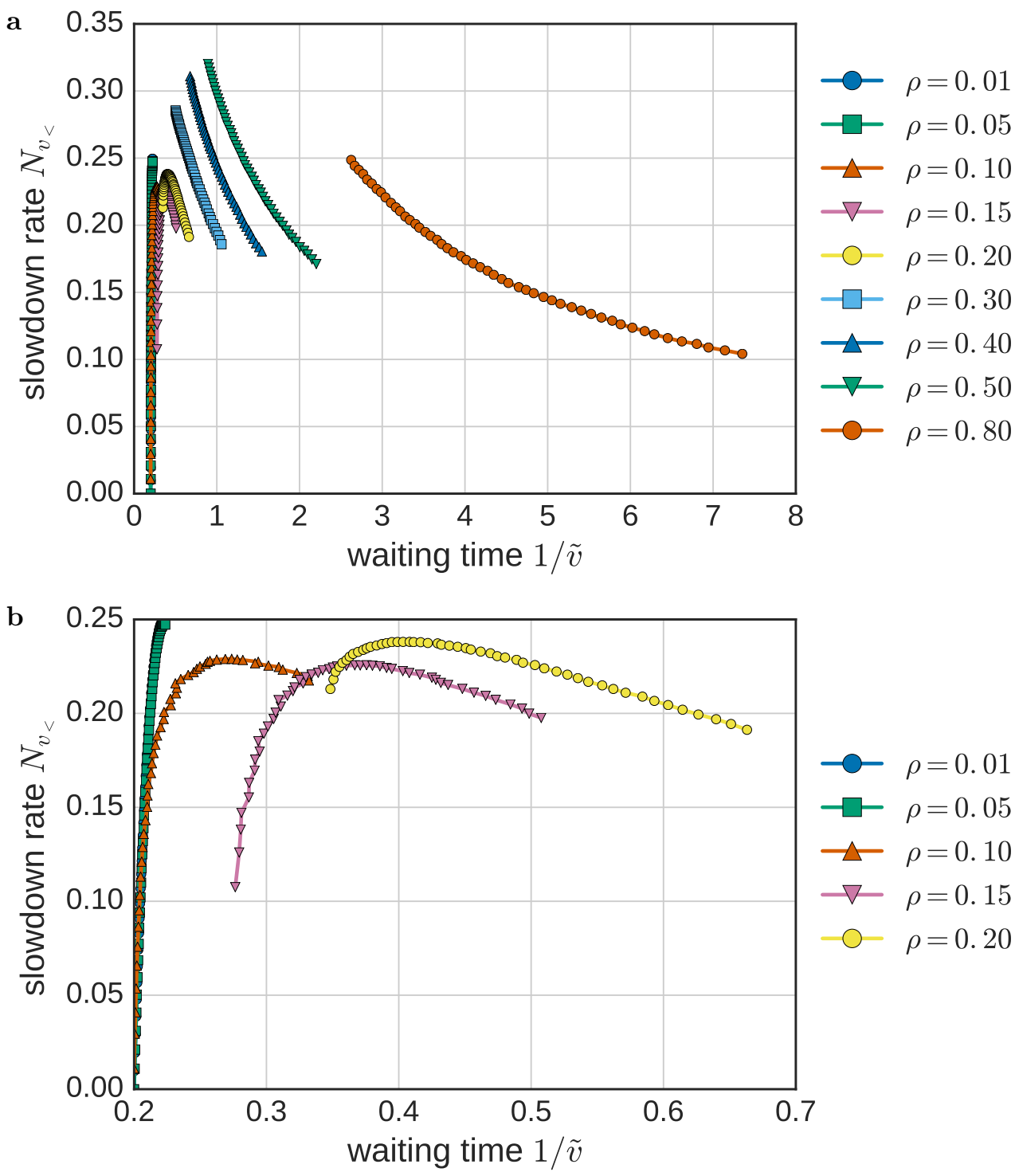

Figure 5: (a) Plots of slowdown incidence vs average waiting time for different densities $\rho=$ $[0.01,0.05,0.1,0.15,0.2,0.3,0.4,0.5,0.8]$. Each point corresponds to a distinct slowdown probability value. (b) We look at densities $\rho<0.3$ and see peaks in the plots for $\rho=[0.1,0.15,0.2]$. All vehicles are motorcycles.

A scenario wherein such transitions driven by high values of $p_{\text {slow }}$ would be that of queuing for parking slots on the side of the road, which is common in places where public parking significantly costs less than in private parking buildings, or where establishments offer free storefront parking. Vehicles that want to park at establishments have higher $p_{\text {slow }}$ while waiting for their turn at parking, which causes a buildup of traffic, effectively pushing density up and inducing a transition from a free flow to an interacting state.

Thus, the slowdown probability $p_{\text {slow }}$ can push even low densities into a region of strong interaction, provided that $p_{\text {slow }}$ is sufficiently high. Extrapolating from the trend, we also see that there is a limit to the maximum possible $\rho$ that can remain in free flow, consistent with theoretical predictions in Sec. 3.2. 


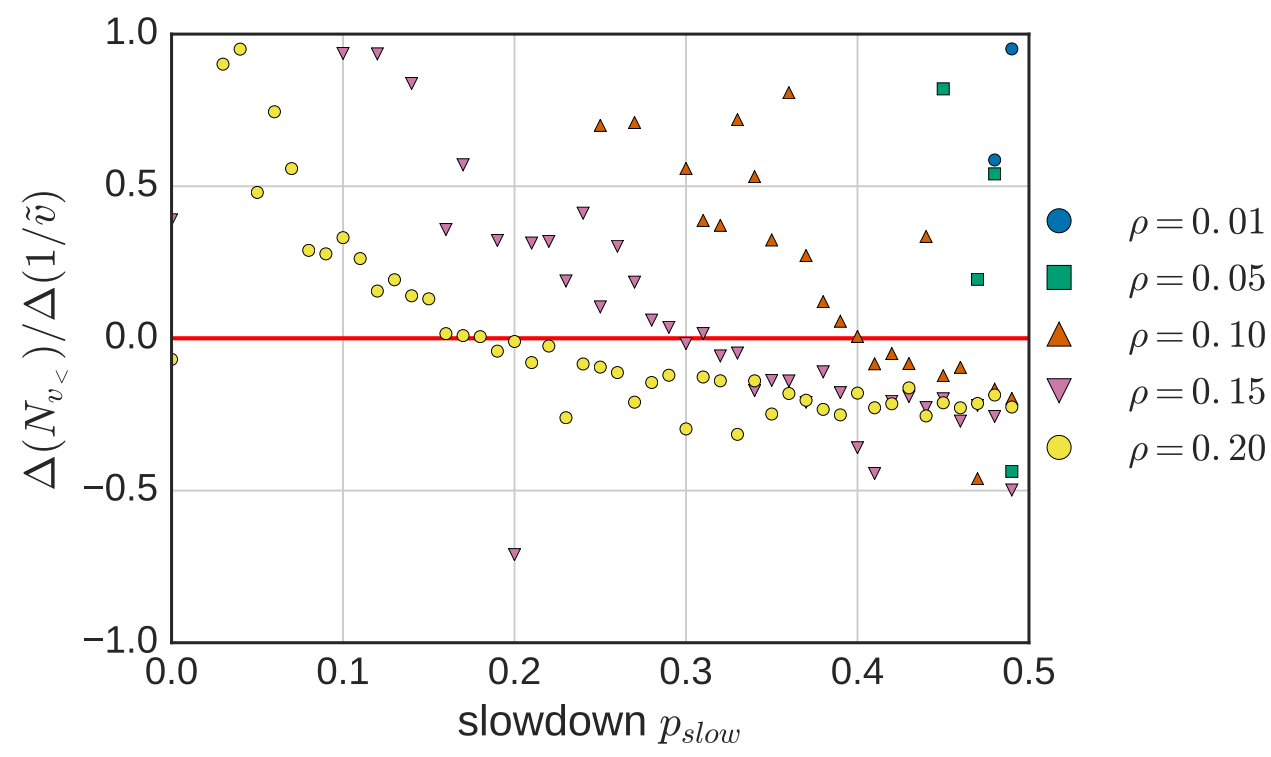

Figure 6: The slopes of the slowdown incidence vs waiting times, plotted against the corresponding slowdown $p_{\text {slow }}$ are shown for $\rho=[0.01,0.05,0.1,0.15,0.2]$. The slope reaches zero for densities $\rho=[0.1,0.15,0.2]$, indicating crossover in the behavior of the slopes.

\section{Summary}

By allowing a fraction $f$ of vehicles to change lanes at a high probability of $p_{\lambda}$, we saw the average speed of vehicles that change lanes was higher than vehicles that do not change lanes. This splitting of the speed distribution only occurs at densities in the interacting region $\rho>\rho_{\text {crit }}$. As the value of $f$ is increased, more vehicles are allowed to change lanes, but we observed that there are diminishing returns in the increase in average speed for vehicles.

The positive benefit of allowing vehicles to change lanes would appear to contradict the common perception that greedy lane changing is detrimental to traffic flow. However, we showed that lane changing is detrimental even when only a small fraction of vehicles change lanes with low $p_{\lambda}$, since this introduces the possibility of lane straddling. Thus, lane changing is potentially beneficial when all vehicles can change lanes while minimizing straddling, but in the case of heavy traffic, not all vehicles can successfully change lanes in a short amount of time.

A theoretical upper bound on the critical density for phase transitions in the absence of lane changing and slowdown behavior was calculated. The critical density is dependent on the maximum speed of vehicles, as well as a vehicle's length. This supports the notion that not only are public utility vehicles more efficient in terms of passenger capacity, public utility vehicles can also lessen inter-vehicular interactions assuming said PUVs are no longer picking up passengers.

We measured the weighted slowdown incidence of vehicles and found a linear dependence of the form $\frac{N_{v_{<}}}{\tilde{v}}=m(\rho, f) p_{\text {slow }}+c(\rho)$, as well as phase transitions in $c(\rho)$ dependent on the density. By relating the weighted slowdown incidence to the average waiting time of vehicles, a crossover in behavior driven by the slowdown probability $p_{\text {slow }}$ was observed, which is closely related to but not equivalent to the density dependent phase transition. This shows that slowdown can influence transitions by pushing even low, free flow densities into states with significant interaction.

\section{Acknowledgment}

The authors acknowledge the support provided by the UPD-OVCRD, University of the Philippines System (CWRG), and Commission on Higher Education-PCARI (IIID-2016-006). 


\section{References}

[1] J. Cramer, A. B. Krueger, Disruptive change in the taxi business: The case of Uber, Am. Econ. Rev. 106 (5) (2016) 177-182. doi:10.1257/aer.p20161002.

[2] Z. Li, Y. Hong, Z. Zhang, Do Ride-Sharing Services Affect Traffic Congestion? An Empirical Study of Uber Entry, SSRN Electron. J. (2016) 1-35.doi:10.2139/ssrn.2838043.

[3] R. Wang, Q. Yuan, Parking practices and policies under rapid motorization: The case of China, Transp. Policy (2013). doi:https://doi.org/10.1016/j.tranpol.2013.08.006.

[4] R. R. Clewlow, G. S. Mishra, Disruptive Transportation: The Adoption, Utilization, and Impacts of Ride-Hailing in the United States, Ucd-Its-Rr-17-07 (October) (2017).

URL https://itspubs.ucdavis.edu/wp-content/themes/ucdavis/pubs/download_pdf.php?id=2752

[5] B. Schaller, Empty Seats, Full Streets. Fixing Manhattan's Traffic Problem, Schaller Consult. December 1 (3) (2017).

[6] H. Hanaura, T. N. Ã, K. Tanaka, Jam formation in traffic flow on a highway with some slowdown sections, Physica A 374 (2007) 419-430. doi:10.1016/j.physa.2006.07.032.

[7] R. Nagai, T. Nagatani, A. Yamada, Phase diagram in multi-phase traffic model, Physica A 355 (2-4) (2005) 530-550. doi:10.1016/j.physa.2005.04.004

[8] M. Bando, K. Hasebe, A. Nakayama, A. Shibata, Y. Sugiyama, Dynamical model of traffic congestion and numerical simulation, Phys. Rev. E. 51 (2) (1995) 1035-1042. doi:10.1371/journal.pcbi.1002329.

[9] Y. Sugiyamal, M. Fukui, M. Kikuchi, K. Hasebe, A. Nakayama, K. Nishinari, S. I. Tadaki, S. Yukawa, Traffic jams without bottlenecks-experimental evidence for the physical mechanism of the formation of a jam, New J. Phys. 10 (2008). doi: $10.1088 / 1367-2630 / 10 / 3 / 033001$.

[10] T. Nagatani, Effect of stoppage time on motion of a bus through a sequence of signals, Physica A 465 (2017) $297-304$. doi:10.1016/j.physa.2016.08.047.

[11] T. Nagatani, Jam formation with line changing at two tollgates on a highway, Physica A 416 (2014) 596-603. doi: $10.1016 / j$.physa.2014.09.024

[12] M. Mohanty, P. P. Dey, Modelling the major stream delay due to U-turns, Transp. Lett. (November) (2017) 1-8. doi: 10.1080/19427867.2017.1401701.

[13] S. Mukherji, S. M. Bhattacharjee, Deadlocks and waiting times in traffic jam, Physica A 245 (3-4) (1997) $534-542$. doi:10.1016/S0378-4371(97)00383-X.

[14] V. Popkov, L. Santen, A. Schadschneider, G. M. Schütz, Empirical evidence for a boundary-induced nonequilibrium phase transition, J. Phys. A. Math. Gen. 34 (6) (2001) L45. doi:10.1088/0305-4470/34/6/103.

[15] A. Schadschneider, Statistical physics of traffic flow, Physica A 285 (2000) 101-120. doi:10.1016/S0378-4371(00) 00274-0.

[16] N. Sugiyama, T. Nagatani, Multiple-vehicle collision in traffic flow by a sudden slowdown, Physica A 392 (8) (2013) 1848-1857. doi:10.1016/j.physa.2013.01.009.

[17] T. Nagatani, S. Yonekura, Multiple-vehicle collision induced by lane changing in traffic flow, Physica A 404 (2014) $171-179$. doi: $10.1016 / j$.physa.2014.02.028.

[18] J. S. L. Combinido, M. T. Lim, Modeling U-turn traffic flow, Physica A 389 (17) (2010) 3640-3647. doi:10.1016/j. physa.2010.04.009.

[19] List of Laws Prescribing Highway Widths, Tech. rep., Department of Public Works and Highways, Manila, Philippines (2006).

URL http://www.dpwh.gov.ph/pdf/issuances/DO/07/34_SIMPLIFIED_GUIDELINES_IROW_CLAIMS/ 12-ListofLawsPrescribingHighwayWidths.pdf

[20] R. Virola, Land Transport in the Philippines: Retrogressing Towards Motorcycles? (2009). URL http://www.nscb.gov.ph/headlines/StatsSpeak/2009/101209_rav_raab_trans.asp

[21] D. N. Dailisan, M. T. Lim, Agent-based modeling of lane discipline in heterogeneous traffic, Physica A 457 (2016) $138-147$. doi: $10.1016 / j$.physa.2016.03.104.

[22] K. Nagel, M. Schreckenberg, A cellular automaton model for freeway traffic, J. Phys. I 2 (12) (1992) 2221-2229. doi: 10.1051/jp1:1992277.

[23] D. Helbing, Traffic and related self-driven many-particle systems, Rev. Mod. Phys. 73 (October) (2001) 1067-1141. doi: 10.1103/RevModPhys.73.1067.

[24] M. Schreckenberg, A. Schadschneider, K. Nagel, N. Ito, Discrete stochastic models for traffic flow, Phys. Rev. E 51 (4) (1995) 2939-2949. doi:10.1103/PhysRevE.51.2939.

[25] H. K. Teoh, E. H. Yong, Renormalization-group study of the Nagel-Schreckenberg model, Phys. Rev. E 97 (3) (2018) 32314. doi:10.1103/PhysRevE.97.032314.

[26] D. A. Rosenblueth, C. Gershenson, A Model of City Traffic Based on Elementary Cellular Automata, Complex Syst. (2011) 305-322.

URL http://www.complex-systems.com/abstracts/v19_i04_a01.html

[27] J. S. L. Combinido, M. T. Lim, Crowding effects in vehicular traffic., PLoS One 7 (11) (2012) e48151. doi:10.1371/ journal.pone. 0048151 . 